\title{
Visual Tracking by Sampling in Part Space
}

\author{
Lianghua Huang, Bo Ma, Member, IEEE, Jianbing Shen, Senior Member, IEEE, Hui He, \\ Ling Shao, Senior Member, IEEE, and Fatih Porikli, Fellow, IEEE
}

\begin{abstract}
In this paper, we present a novel part-based visual tracking method from the perspective of probability sampling. Specifically, we represent the target by a part space with two online learned probabilities to capture the structure of the target. The proposal distribution memorizes the historical performance of different parts, and it is used for the first round of part selection. The acceptance probability validates the specific tracking stability of each part in a frame, and it determines whether to accept its vote or to reject it. By doing this, we transform the complex online part selection problem into a probability learning one, which is easier to tackle. The observation model of each part is constructed by an improved supervised descent method and is learned in an incremental manner. Experimental results on two benchmarks demonstrate the competitive performance of our tracker against state-of-the-art methods.
\end{abstract}

Index Terms - Visual tracking, part space, sampling.

\section{INTRODUCTION}

G IVEN a specified object in the first frame, the task of visual tracking is to locate it in the successive video frames. As a fundamental topic in computer vision, object tracking plays an important role in numerous applications such as visual surveillance, humancomputer interaction and augmented reality. Despite decades of studies [1], [35], [41], [46], visual tracking is still a challenging task due to target appearance variations such as object deformation, occlusion, illumination changes, motion blur and background clutters.

For object tracking, local appearance models [5], [7], [8] are generally more robust than holistic ones [4], [6], since many challenging factors, e.g., the object deformation and

This work was sup- ported in part by the National Natural Science Foundation of China under Grant 61472036, in part by the National Basic Research Pro- gram of China (973 Program) under Grant 2013CB328805, and in part by the Australian Research Council's Discovery Projects Funding Scheme under Grant DP150104645. Specialized Fund for Joint Build- ing Program of the Beijing Municipal Education Commission. The asso- ciate editor coordinating the review of this manuscript and approv- ing it for publication was Prof. $\mathrm{Ce}$ Zhu. (Corresponding author: Bo Ma.)

L. Huang, B. Ma, J. Shen, and H. He are with the Beijing Laboratory of Intelligent Information Technology, School of Computer Science, Beijing Institute of Technology, Beijing 100081, China (e-mail: bma000@bit.edu.cn; shenjianbing@bit.edu.cn).

L. Shao is with the School of Computing Sciences, University of East Anglia, Norwich NR4 7TJ, U.K. (e-mail: ling.shao@ieee.org).

F. Porikli is with the Research School of Engineering, The Australian National University, Canberra, ACT 0200, Australia (e-mail: fatih.porikli@anu.edu.au). partial occlusions, can be viewed as local noise or variations. Numerous local appearance models have been proposed in recent years and have achieved promising results. Existing methods can be roughly categorized into the following classes: sparse representation based methods [7]-[9], [22], segmentation based methods [5], [26], [29], pooling methods [10], [11] and part-based methods [12], [20], [44].

Sparse representation based methods work under the assumption of sparse noise (e.g., partial occlusions and local background clutter), and represent the target as a sparse combination of templates and noisy pixels [8], [9]. Despite their effectiveness in handling occlusions and background noise, they are not suitable for tackling deformable objects, where the shifted parts will be mistakenly regarded as noise. Segmentation based methods [5] separate the target and the background into several irregular patches (e.g., superpixels), and formulate tracking as an online segmentation or patch classification problem. The flexibility of these methods makes them handle partial occlusions and object deformation robustly. However, it is still difficult for them to obtain accurate bounding boxes. Besides, the segmented patches are not uniform in size, which makes them difficult to generalize. Pooling methods [10], [11], [43] obtain local patches from the target by performing sliding windows on it, and represent the target with pooled features of local descriptors. These methods can decrease the impact of local noise. Nevertheless, when variations of large areas exist, such as object deformation and severe occlusions, the noisy blocks will have negative impact on target locating.

When an object deforms or suffers from occlusions, its holistic appearance changes a lot, but part of its local appearance remains identifiable. Based on this idea, partbased models have been introduced in many computer vision tasks [12], [14]-[17], [44]. In object detection [45], [47], deformable part models (DPM) [14] has been proposed and achieved state-of-the-art performance. Subsequently, it attracts popularity and numerous improvements to DPM have been presented [15]-[17]. In visual object tracking, part-based models have also been proposed to deal with target deformation and partial occlusions [12], [20], [44], [48]. Yao et al. [12] presented a part-based tracking method with online latent structured learning. This work can be viewed as an online extension of DPM in visual tracking. In [44], a part-based tracking method with cascaded regression was proposed, which exploits the spatial constraints between parts to learn the intrinsic shape of an object. Lu et al. [20] proposed an online tracking-learning-parsing framework that utilizes an and-or graph to capture the construction of objects. 


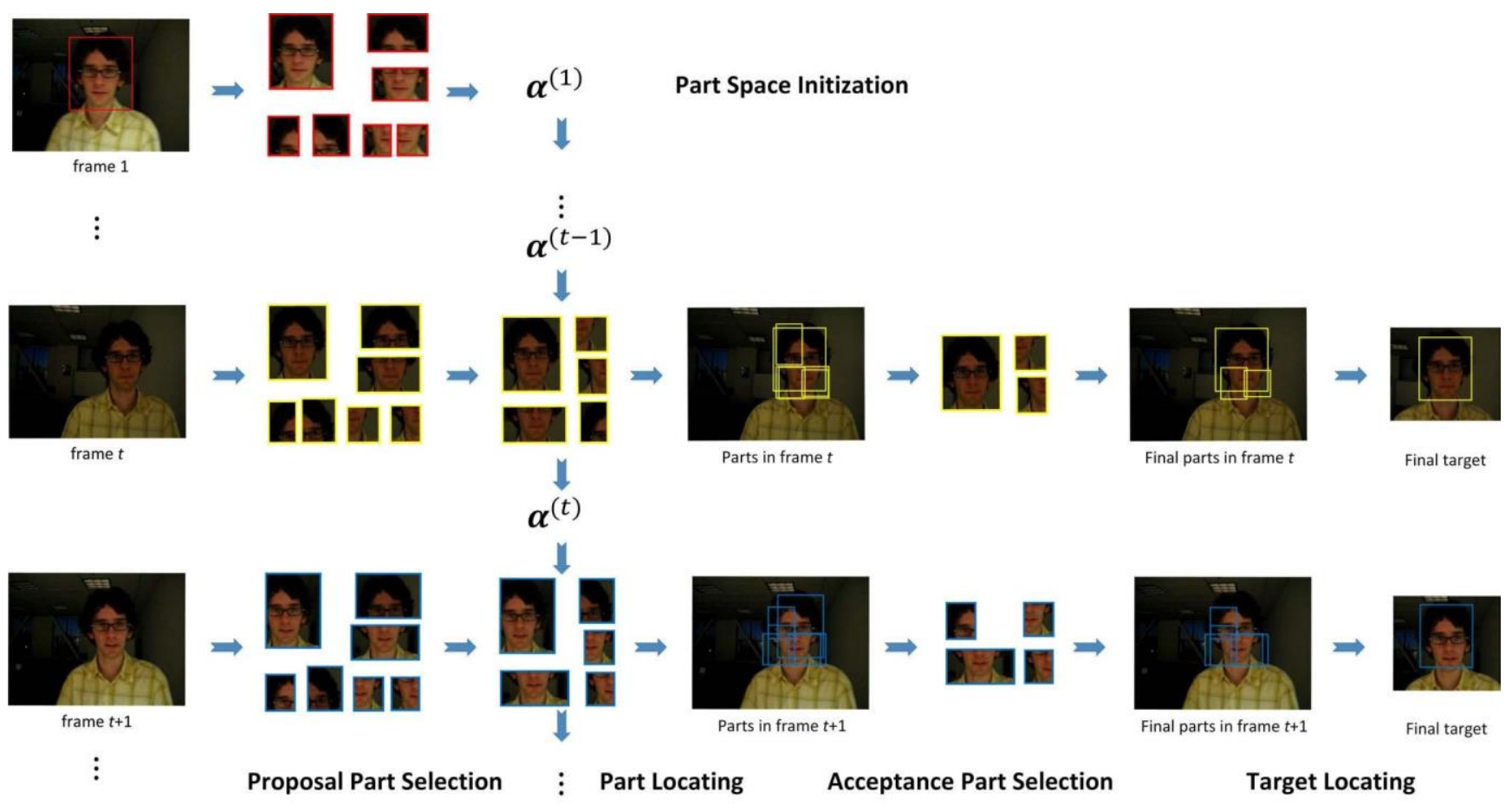

Fig. 1. Illustration of the proposed part-based tracking algorithm. A part space of the target is initialized in the first frame according to the proposal distribution $a$. Then $a$ is updated in each frame based on the contributions of parts to target locating. We sample parts according to $a$ and track them independently. Votes of different parts are accepted/rejected according to an acceptance probability $\beta$, then the target location is estimated based on the accepted votes.

Although above trackers have made attempts to apply the part-based strategy in visual tracking, the part-based methods for tracking are far less popular than for object detection. One of the main reasons is the lack of training samples with the tracking data. For object detection, there are enough samples for determining the best way of part separation. However, for object tracking, the only information provided is the target location in the first frame. It is difficult to determine how the target is separated with only one sample of an object. A better way is to learn the separations online. However, an online part separation model is usually complex and time consuming.

We propose a new part-based method to solve the above issues from the perspective of probability sampling. The overview of our method is illustrated in Fig. 1. We represent each target by a part space, which contains sufficient regions to cover most structures of objects, and two online learned probabilities on it - the proposal distribution $\boldsymbol{\alpha}$ and the acceptance ratio $\boldsymbol{\beta}$. The $\boldsymbol{\alpha}$ represents the historical information of different parts and is applied on the first round of part selection, while the $\boldsymbol{\beta}$ validates the frame specific tracking stability of each part and determines whether to accept a part's vote to the target location or not. Thus, the complex online part selection problem is transformed into a probability learning one, which is much easier to solve. The observation model of each part is constructed by an improved supervised descent method (SDM) [18], where we incorporate the basic SDM model with a confidence evaluation scheme for indicating the reliability of each predicted descent direction. We propose an incremental cascaded support vector regression (ICSVR) algorithm for model updating. To recover the unselected parts, we further present a part relocating scheme. Our source code will be available online. ${ }^{1}$

Compared to the existing approaches, the proposed visual tracking method provides the following contributions:

- We propose a novel part-based method, which represents each target by a part space and two learned probabilities, to transform the complex online part selection problem into a probability learning one.

- An improved supervised descent method (SDM) is proposed to construct the observation model of each part, which incorporates the basic SDM model with a confidence evaluation scheme for indicating the reliability of each predicted descent direction.

- To achieve robust visual tracking, we further propose an incremental cascaded support vector regression (ICSVR) algorithm for model updating and an unselected relocating scheme for parts updating.

\section{RELATED WORKS}

In this section, we briefly review three closely related topics: part-based models, sampling based tracking methods and the supervised descent methods.

\section{A. Part-Based Models}

Partial occlusions, background noise and object deformation are some of the most common phenomena in real world

\footnotetext{
${ }^{1}$ http://github.com/shenjianbing/partspacetrack
} 
videos, and they also cast a challenge for vision tasks such as object detection, recognition and visual tracking. When occlusions or deformation occur, the global appearance of an object may vary largely, but the local appearance usually remains identifiable. Based on this observation, several notable part-based methods have been proposed.

One notable work is the deformable part models (DPM) [14] proposed in the area of object detection. In this method, objects are represented as discriminatively trained deformable part models, and the non-convex training problem is solved by a latent SVM algorithm. Attracted by the performance and the extensibility of DPM, several extensions and variants have been proposed in [15]-[17] and [19]. In visual object tracking, part-based models have also been introduced to deal

with local variations. Yao et al. proposed an online extension of DPM for tracking non-rigid objects. It represent an object as a feature vector composed of part feature vectors and part offsets, and cast tracking as an online latent SVM learning problem. It shows better performance compared to its counterpart [4]. Lu et al. presented a tree-structured model to represent the part configurations and introduced a trackinglearning-parsing framework to perform online object tracking.

In [49], a multiple part tracking framework was proposed based on the KCF [6] tracker to achieve real-time performance.

A closely related work to ours is the TRIC algorithm [44].

Both TRIC and our work are part-based tracking models and construct the observation model based on the supervised descent method (SDM) [18]. However, the differences between them are obvious. First, our method aims at learning the best way of part selection, while TRIC is conducted to build a shape model for a target and it performs no part selection. Second, our method tracks each part independently and combine their results in postprocessing steps. Instead, TRIC locates each part based on its three adjacent parts. Third, we have improved the SDM by introducing an confidence evaluation scheme.

\section{B. Sampling Based Tracking Methods}

Sampling based methods are widely used when the cost function is non-convex and when the searching space is large. Several tracking by sampling methods have been proposed [38], [39] to efficiently optimize for better models. In [38], different tracking algorithms are decomposed into four ingredients: appearance and motion models, state rep-

resentation types and observation types. These ingredients are sampled iteratively by using the Gibbs sampling strategy to

generate several trackers. Then, the accepted state having the highest posterior likelihood is chosen as the final tracking result. Hong and Han [39] present an offline tracking method by reorganizing the sequential video frames in a tree-structured graph. It finds the optimal tree that minimizes the tracking costs along the paths from root to leaf nodes by using the Markov Chain Monte Carlo (MCMC) based sampling method. Then, a probabilistic test is performed on the tree to determine whether to accept it or not, and the optimal solution can be obtained from the accepted trees.

Both the above two approaches and our method treat tracking as a probability sampling process, and the proposal/acceptance steps are adopted to obtain the optimal solution. As compared with their methods by sampling trackers or frame organizations, our method regards each tracking object as a configuration in its part space and searches for an optimal part configuration by sampling to improve tracking.

\section{Supervised Descent Methods}

The Supervised Descent Method (SDM) [18], [27] is originally applied to facial landmark detection. Due to its extensibility, it has been widely applied to many other areas, including 3D pose estimation [25] and visual tracking [44]. Many basic vision problems can be formulated as a Nonlinear Least Squares (NLS) problem: $\min _{\mathbf{x}} f(\mathbf{x})=x h(\mathbf{x})-\boldsymbol{\varphi} z_{2}$ where

$\boldsymbol{\varphi}_{*}$ is a template, $\mathbf{x}$ is a state (location, angle, etc.) variable and $h()$ is a feature extractor. Since most feature extractors $h$ ( ) are not twice differentiable, the idea of SDM [18] is to learn the mapping from features to descent directions by linear cascaded regressions, instead of calculating the Jacobian and Hessian matrices in Newton's method.

Despite the effectiveness of SDM, one of its main drawbacks is that it only estimates descent directions, and does not output values on how reliable the estimations are. This paper addresses the issue of confidence evaluation in SDM.

\section{BASIC TRACKER}

In the proposed part-based method, each part is tracked with an independently learned observation model. This section presents details on the basic tracking approach for each part.

\section{A. Cascaded Regression}

In our approach, the observation model for each part is constructed based on the supervised descent method (SDM) [18], which learns the nonlinear projection from features to descent directions in a cascaded linear manner. Specifically, for a part located at $\mathbf{v}=(x, y) \in \mathrm{R}^{2}$, where $(x, y)$ denotes the central coordinate, we draw samples $\left\{\mathbf{v}_{i}\right\}_{i=1}^{n}$ around $\mathbf{v}$ to obtain training data $\left\{\left(\sigma \mathbf{v}_{i}, \boldsymbol{\varphi}_{i}\right)\right\}$, where $\boldsymbol{\varphi}_{i} \in \mathrm{R}^{p}$ denotes the extracted feature of sample $i$ and $\sigma \mathbf{v}_{i}=\mathbf{v}-\mathbf{v}_{i}$ is its offset to the groundtruth. The SDM learns the projection matrices $\left\{\mathbf{R}_{k} \in \mathrm{R}^{2 \times}{ }_{k}\right\}_{1}^{C}$ in a cascaded way by iteratively optimizing the following $C$ problems [18]:

$$
\begin{aligned}
& \underset{\mathbf{R}_{k}}{\min _{i=1}}{ }_{i}^{\pi} \times \mathbf{v}^{k} \mathbf{R}_{k} \boldsymbol{\varphi}_{i} \times{ }_{2}+\lambda \times \mathbf{R}_{k} \times{ }_{2}, \\
& \mathbf{v}_{i}^{k+1}=\mathbf{v}_{i}^{k}+R_{k} \boldsymbol{\varphi}_{i}^{k},
\end{aligned}
$$

where $k=1, \cdots, C$ denotes the cascade index, $\underset{i}{\mathbf{v}}=\mathbf{v}_{i}, \underset{i}{\boldsymbol{\varphi}^{k}}$ is the feature extracted at $\mathbf{v}_{i}^{k}$ and $\lambda$ is a regularization parameter for controlling the model complexity.

In our method, we use the Support Vector Regression (SVR) algorithm instead of (1) for learning the projections $\boldsymbol{R}_{k} \xi_{k=1}$ for two reasons. First, the support vectors in SVR preserve historical information and can facilitate model updating. As well, they can also largely avoid the model being deteriorated by tracking failures. Second, the SVR is less vulnerable to noise, which largely exists when linear models are used to model nonlinear relationships. Let $\mathbf{r}^{(k j)}, j=1,2$, denotes the $j$ th row 
of $\mathbf{R}_{k}, \sigma v_{i}^{(k j)}$ denotes the $j$ th entry of $\sigma \mathbf{v}^{k}$, and the cascaded $\mathrm{SVR}$ is then formulated as:

$$
\begin{aligned}
& \left.\min _{\mathbf{r}(k j), \xi(k j), \boldsymbol{\xi}^{*(k j)}} \overline{2}^{-} \times \mathbf{r}^{(k j)}\right)_{X_{2}+\eta_{1}}^{\underline{n}}\left(\xi_{i}+\xi_{i}\right), \\
& \text { s.t. }\left(\mathbf{r}^{(k j)} \cdot \boldsymbol{\varphi}^{k}\right)-\sigma v^{(k j)} \stackrel{i=1}{\leq} \varepsilon_{1}+\xi^{(k j)} \text {, }
\end{aligned}
$$

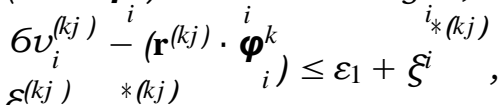

$$
\begin{aligned}
& \zeta_{i}, \xi_{i} \geq 0 \text {, } \\
& i=1, \cdots, n, k=1, \cdots, C,
\end{aligned}
$$

where $\eta_{1}$ is a regularization factor, $\xi_{i}^{(k j)}, \xi_{i}^{*(k j)}$ are slack variables and $\varepsilon_{1}$ is a pre-set margin. We set $\varepsilon_{1}=5$, which means the allowed prediction bias without punishment is 5 pixels.

\section{B. Confidence Evaluation}

Despite the effectiveness of SDM, its main drawback is the lack of a mechanism for indicating how reliable an offset prediction is. In this section, we present a confidence evaluation scheme for SDM.

In the training stage, if one regress iteration pulls a sample closer to the groundtruth, we say that the sample is more credible and vice versa. Based on the idea, we propose to learn an extra set of projection matrices $\left\{\mathbf{Q}_{k} \in \mathrm{R}^{1 \times p}\right\}^{C}$ for confidence evaluation. We take the ratio of overlap rates before and after regression $\dot{\theta}_{i}^{k}=\left(o_{i}^{k+1}\right)^{2} / o_{i}^{k}$ (where $o_{i}^{k}$ denotes the overlap between $\mathbf{v}^{k}$ and $\left.\mathbf{v}\right)$ as the label to train $\left\{\mathbf{Q}_{k}\right\}^{C} \underset{k=1}{:}$

$$
\begin{aligned}
& \min _{\mathbf{Q}_{k}, \xi^{(k), \xi^{*}(k)}} 2^{-} \times \mathbf{Q}_{k} \times_{2}+\eta_{2} \stackrel{n}{-}\left(\xi_{i}^{(k)}+\xi_{i}^{*(k)}\right), \\
& \begin{array}{cc}
\text { s.t. } & \mathbf{Q}_{k} \cdot \boldsymbol{\varphi}_{i}^{k}-\theta_{i_{k}}^{k} \leq \varepsilon_{2}+\xi_{i}^{i=1} \\
\theta^{k} & { }_{i k}^{(k)},
\end{array} \\
& \xi^{i}(k) \mathbf{Q}_{k} \cdot \boldsymbol{\varphi}_{i} \leq \varepsilon_{2}+\xi^{*}{ }_{i} \\
& { }_{i}, \xi_{i} \geq 0 \text {, } \\
& i=1, \cdots, n, k=1, \cdots, C \text {. }
\end{aligned}
$$

We set $\varepsilon_{2}=1$, which is comparable with the magnitude of $\theta_{i}{ }^{k}$. During testing, with the estimated $\left\{\hat{\theta}^{k}=\mathbf{Q}_{k} \cdot \boldsymbol{\varphi}^{k}\right\}_{i}^{C}$ for each ${ }^{i}$ sample, the credibility $c_{i}$ is then computed as:

$$
c_{i}={ }_{k=1}^{C} \hat{\theta}_{i}^{k}, \quad k=1, \cdots, C .
$$

\section{Part Locating}

The motion model of our method is based on the particle filters framework [13]. When locating a part in a new frame, we sample around its last estimated position $\mathbf{v}$ from Gaussian distribution $N\left(\mathbf{v}, €_{2}\right)$, where $€_{2}=\operatorname{diag}\left(r_{2}, r_{2}\right)$, to obtain

$m$ candidates $\left\{\mathbf{v}_{i}, \boldsymbol{\varphi}_{i}\right\}_{=1}^{m}$. With the learned cascaded model, we iteratively pull each sample $\mathbf{v}_{i}$ to the estimated part location from a start state $\mathbf{v}_{i}^{1}$ :

$$
\stackrel{\mathbf{v}}{k+1}_{i}=\mathbf{v}_{i}^{k}+\mathbf{R}_{k} \boldsymbol{\varphi}_{i}^{k}, k=1, \cdots, C,
$$

After $C$ iterations, we obtain all the estimated states $\hat{\mathbf{v}}_{i}=\mathbf{v}^{C}$

+1 . Intuitively, the most densely voted location is more likely to be the part location.
In our method, the dominant set algorithm [28] is adopted to seek for the voting center. The dominant set algorithm computes the weight $w_{i}$ for each sample by optimizing:

$$
\begin{aligned}
& \max _{\mathbf{w}} \mathbf{w}^{\mathrm{T}} \mathbf{A} \mathbf{w}, \\
& \text { s.t. } \mathbf{w} \in a,
\end{aligned}
$$

where $a=\left\{\mathbf{w} \in \mathrm{R}^{m}: \mathbf{w} \geq \mathbf{0}\right.$ and $\left.\mathbf{e}^{\mathrm{T}} \mathbf{w}=1\right\}$, e $\in \mathrm{R}^{m}$ is a vector of all 1s, $\mathbf{A} \in \mathrm{R}_{m \times 2 m}$ is an affinity matrix with each entry $A_{i j}=\exp \left(-\frac{x \hat{\mathbf{v}}_{i}-\hat{\mathbf{v}}_{j} x_{2}}{2 \sigma_{A}^{2}}\right)$ representing the similarity between $\hat{\mathbf{v}}_{i}$ and $\hat{\mathbf{v}}_{j}, \sigma_{A}$ is a scaling factor. As noted in [28], $\sigma_{A}$ is set to be the median value of all entries in A. Finally, the part is located by:

$$
\hat{\mathbf{v}}={ }_{i=1}^{m} w_{i} \hat{\mathbf{v}}_{i} .
$$

Taking the sample confidence $c_{i}$ into consideration, we slightly modify the affinity matrix $\mathbf{A}$ as:

$$
A_{i j}^{*}=c_{i} \cdot c_{j} \cdot A_{i j} .
$$

The rest of the voting process is the same as described before.

\section{Updating Scheme}

To adapt the basic model to part appearance variations, we propose an Incremental Cascaded Support Vector Regression (ICSVR) algorithm for model updating. To deduce the updating scheme, we first investigate the relationship between Support Vector Classification (SVC) and Support Vector Regression (SVR). With training data $\left\{\mathbf{x}_{i}, y_{i}\right\}_{i=1}^{h}$, the SVR problem can be formulated as:

$$
\begin{gathered}
\min _{\mathbf{w}, \xi, \xi^{*}} \sum^{1} \times \mathbf{w} \times_{2}+\eta^{-}\left(\xi_{i}+\xi_{i}^{*}\right), \\
\text { s.t. }(\mathbf{w} \cdot \underset{i}{\mathbf{x}})-\underset{i}{y} \stackrel{i=1}{\leq} \varepsilon+\xi_{i}, \\
y_{i}-\left(\mathbf{w} \cdot \mathbf{x}_{i}\right) \leq \varepsilon+{ }_{i} \xi^{*}, \\
\xi_{i}, \xi_{i}^{*} \geq 0, i=1, \cdots, h,
\end{gathered}
$$

where $\eta$ is a regularization parameter, $\varepsilon$ is a pre-set margin and $\xi_{i}, \xi_{i}^{*}$ are slack variables.

The above problem is equivalent to a Support Vector Classification model formulated on the modified training data $\left\{\left(\mathbf{z}_{i}, 1\right)\right\}_{i=1}$ and $\left\{\left(\mathbf{z}_{i},-1\right)\right\}_{i=h+1}$, where $\mathbf{z}_{i}=\left(\mathbf{x}_{i}, y_{i}+\varepsilon\right)$ for $i=1, \cdots, h$ and $\mathbf{z}_{i}=\left(\mathbf{x}^{\mathrm{T}}, y_{i}-\varepsilon\right)^{\mathrm{T}}$ for $i=h+1, \cdots, 2 h$ :

$$
\begin{aligned}
& \min _{\mathbf{w}, \boldsymbol{\xi}} \frac{1}{2} \mathbf{x}^{2} x_{2}+\eta_{i=1}^{2 h} \xi_{i}, \\
& \text { s.t. } \left.\left(\mathbf{w} \cdot z_{i}\right) \geq 1-\xi_{i}\right) \geq 1-\xi_{j}, i=1, i=h+1, \cdot h, 2 h \text {, } \\
& \xi_{i} \geq 0, i=1, \cdots, 2 h,
\end{aligned}
$$

where $\eta$ is a regularization parameter.

In this case, the online learning of SVR can be implemented by online SVC algorithms with slightly modified training data. We use the twin prototypes algorithm [30] in [3] as the SVC updater in our approach. In the twin prototypes algorithm, the SVC model can be compactly summarized as a prototype 
set $\left\{\psi_{i}, S_{i}, s_{i}\right\}_{=1}^{B}$, where $\psi_{i}$ is a feature vector, $S_{i}$ is a binary label and $s_{i}$ is a counting number that indicates how many support vectors are represented by this instance. With new data $\left\{z_{j}, Y_{j}\right\}_{j=1}^{J}$ where $z_{j}$ is a feature vector and $Y_{j}$ is a binary label, the SVC model is updated by minimizing:

$$
\begin{aligned}
\left.\min _{\mathbf{w}, b} \frac{1}{2} \times \mathbf{w} \times 2+K{\left(\frac{1}{B}{ }_{i=1}^{\underline{B}} s_{i} L_{h}(S i\right.}_{w_{i}} \psi_{i} ; \mathbf{w}\right) \\
\left.+\frac{1}{J}_{j=1}^{\underline{J}} L_{h}\left(z_{j}, V_{j} ; \mathbf{w}\right)\right)
\end{aligned}
$$

where $L_{h}$ is the hinge loss.

After training, support vectors from the new data are added to the prototype set with counting number 1 . When the size of the prototype set is larger than a predefined budget $\vec{B}$, the pair of prototype instances of the same label with the mimimal distance are merged into $\left(\psi^{*}, S^{*}, s^{*}\right)$, where

$$
\psi^{*}=\frac{s_{i_{1}} \psi_{i_{1}}+s_{i_{2}} \psi_{i_{2}}}{s_{i_{1}}+s_{i_{2}}}, \quad S^{*}=S_{i_{1}}, s^{*}=s_{i_{1}}+s_{i_{2}} .
$$

In our experiments, we use $\hat{B}=80$ as the budget and $K=100$ for weighting the loss term, though we found that our tracking performance tends to be insensitive to these settings.

Finally, we extend the online SVR to the cascaded version. After tracking in each frame, we draw samples $\left\{\sigma^{1}{ }_{i}^{1} \boldsymbol{\varphi}_{i}^{1}\right\}{ }_{i=1}$ around the estimated part location $\mathbf{v}$ from Gaussian distribution $N\left(\mathbf{v}, €_{1}\right)$, where $€_{1}=\operatorname{diag}\left(r_{1}, r_{1}\right)$, to obtain training data for the first cascade. Then each sample is iteratively updated as:

$$
v^{k+1}=v_{i}^{k}+R_{k} \varphi_{i}^{k} .
$$

The samples $\left\{\sigma_{\mathbf{v}_{i}^{k}}^{k}, \boldsymbol{\varphi}_{i}^{k}\right\}_{i=1}^{I}, k=2, \cdots, C$ are then collected for the updating of the $k$ th cascade, where $\quad \sigma \mathbf{v}^{k}=\mathbf{v}-\mathbf{v}_{i}^{k}$.

\section{TRACKING By SAMPling IN PART SPACE}

As described in Section III, the observation model for each part is represented by a set of projection matrices $\left\{\mathbf{R}_{k}, \mathbf{Q}_{k}\right\}_{1}{ }_{1}^{C}$. This section presents details on the online selection and updating of these parts, and how to use them for target locating.

\section{A. Part Space}

In our implementation, the initial parts are automatically generated based on the bounding box (x,y, width, height) of the tracking target in the first frame. Specifically, we separate the bounding box into two parts equally along the long side. For each part, we perform the same partition process to obtain another pair. After $P$ iterations, $L=-{ }^{-} 2^{c}$ parts are obtained. We set $P=2$ in our experiments, which generates $L=7$ parts (as illustrated in Fig. 2). These parts make up the 'part space' in our approach. Since the main idea of our approach is to transform the complex online part selection problem to a probability learning one, the roughly selected regions are enough for it to work well. Though, we believe our method can be easily extended with automaticinitial parts, such as region proposal for the initial bounding boxes. During tracking, a probability $\boldsymbol{\alpha} \in \mathrm{R}^{L}$ on the part space is learned online to memorize the contributions of different parts, which

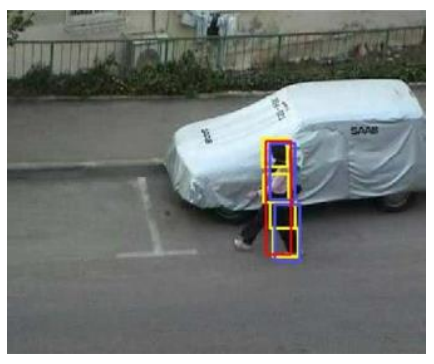

(a)

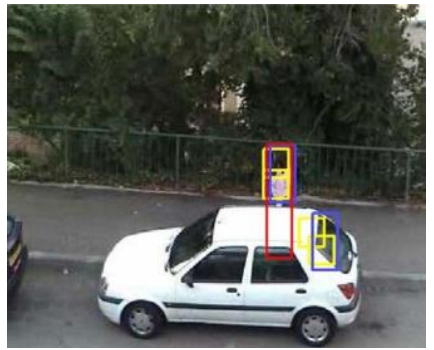

(c)

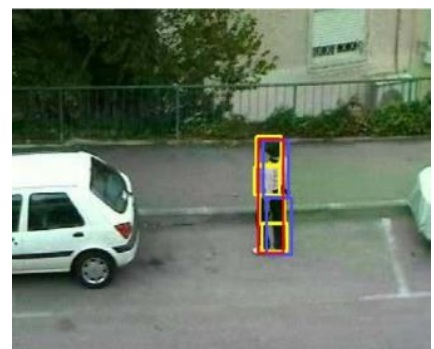

(b)

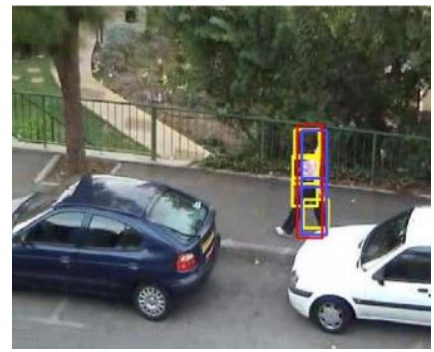

(d)
Fig. 2. Illustration of part space in sequence Woman. For clarity, we only show the parts that are no bigger than half of the object size. Boxes in blue and yellow denote parts of different sizes. The red boxes denote the tracking results. (a) Part space in frame \#16. (b) Part space in frame \#70. (c) Part space in frame \#128. Due to occlusion, the bottom blue and yellow parts drift away from the target. (d) The occluded parts are relocated in frame \#168.

is served as a proposal distribution and ${ }_{l=1}^{-} a_{l}=1$. When locating the target, we first sample $L_{a}=5$ parts from the part space according to $\boldsymbol{\alpha}$ without replacement, and then track each one independently with its observation model.

After tracking, the confidences for different parts are obtained and normalized to calculate the acceptance ratio $\boldsymbol{\beta} \in \mathrm{R}^{L}$ where $\beta_{l} \in[0,1]$. The $\boldsymbol{\beta}$ examines the tracking result of each part in the current frame and determines whether to accept its vote to the target location.

With the accepted parts, the target is located with their votes by using the dominant set algorithm. The online learning of probabilities $\boldsymbol{\alpha}$ and $\boldsymbol{\beta}$ and the relocating of unaccepted parts are described in the following sections.

\section{B. Part Selection}

1) Proposal Distribution: The proposal distribution $\boldsymbol{\alpha} \in \mathrm{R}^{L}$ evaluates the contributions of different parts over time and is used for the first round of part selection. Denote $\hat{\mathbf{s}}$ as the estimated target location in a frame and $\hat{\mathbf{s}}_{l}, l=1, \cdots, L$ as the votes from parts. We define the contribution $g_{l}$ of a part to target locating as:

$$
g_{l}=\exp \left(-\frac{\times \hat{\mathbf{s}}_{l}-\hat{\mathbf{s}}^{\times_{22}}}{2\left(\sigma_{1}\right)^{2}}\right),
$$

where $\sigma_{1}$ is the scaling factor and is set to 4 pixels, which is comparable with the allowed prediction bias.

The normalized contribution vector $\overline{\mathbf{g}}$ is calculated as: $\bar{g}_{l}=$ $\frac{g_{l}}{-\mathbb{L}=1 g_{l}}$ so that $\quad l_{l=1} \bar{g}_{l}=1$. The initial $\boldsymbol{\alpha}^{(1)}$ is set as the normalized part areas:

$$
a_{l}^{(1)}=\frac{S_{l}}{-L_{l=1} S_{l}},
$$




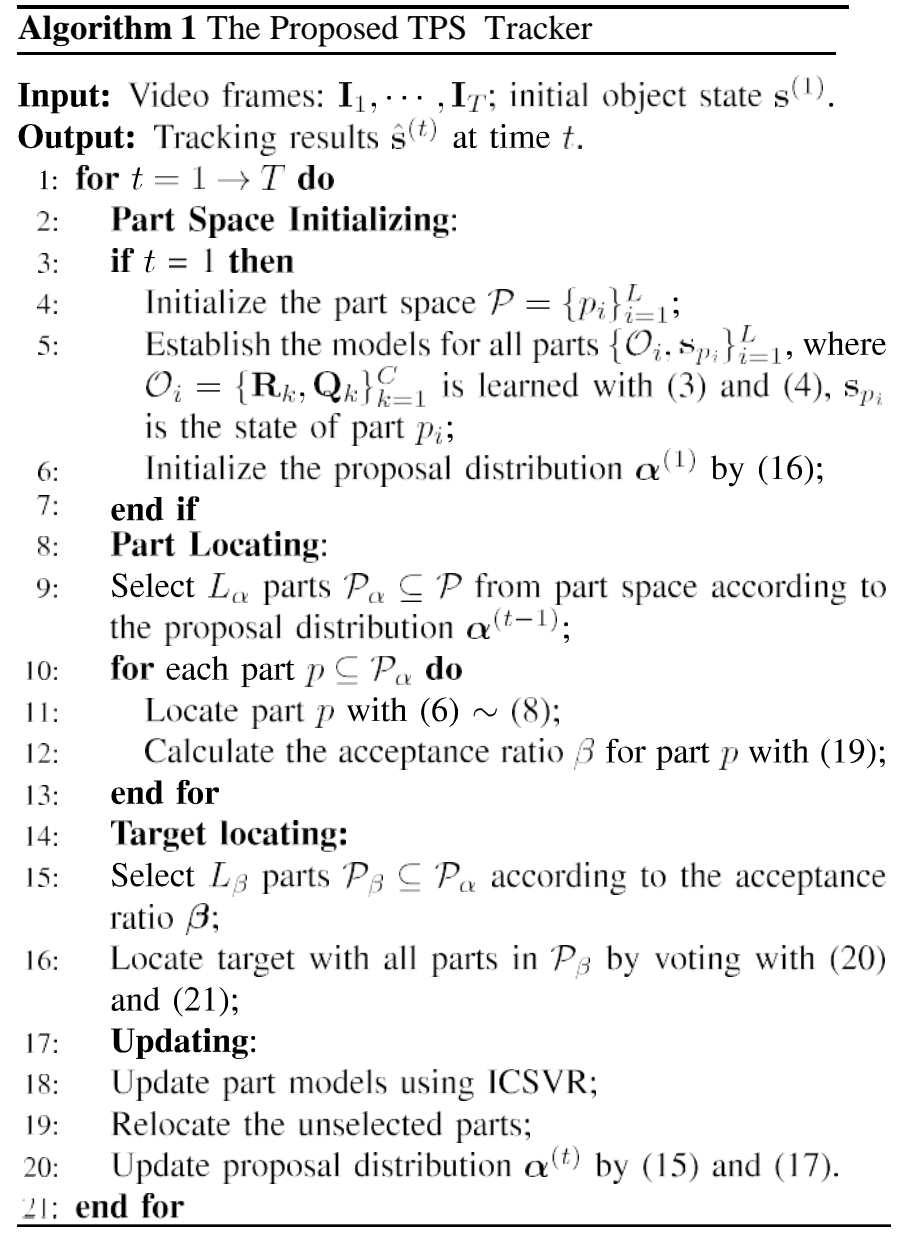

where $S_{l}$ denotes the area of part $l$ and the superscript (1) denotes the frame index. This is consistent with the intuition that larger parts are more recognizable.

Afterwards, the $\boldsymbol{\alpha}^{(t)}$ is updated as:

$$
\boldsymbol{\alpha}^{(t)}=\mu \boldsymbol{\alpha}^{(t-1)}+(1-\mu) \overline{\mathbf{g}}^{(t)}, \quad t=2, \cdots, T,
$$

where $\overline{\mathbf{g}}^{(t)}$ is the contribution vector in the $t$-th frame and $\mu$ is a forgetting factor fixed at 0.9 in our experiments.

2) Acceptance Probability: The probability $\beta_{l} \in[0,1]$, $l=1, \cdots, L$ emphasizes the frame specific tracking performance of a part, which is served as an acceptance ratio. The basic observation is, if a part is being occluded or disturbed by background noise, its candidate votes (see Section III-C) will be scattered, otherwise densely distributed. Based on this idea, we define the voting stability $\tau$ for each part as:

$$
\tau={ }_{i=1}^{n} c_{i} \exp \left(-\frac{\times \hat{\mathbf{v}}_{i}-\hat{\mathbf{v}} \mathrm{x}_{2}}{2\left(\sigma_{2}\right)^{2}}\right),
$$

where $\hat{\mathbf{v}}$ is the voting center, $\hat{\mathbf{v}}_{i}$ denotes the estimation of the $i$ th candidate (see Section III-C), $c_{i}$ is the confidence value as described in Section III-B and $\sigma_{2}$ is a scaling factor fixed to 3 pixels, which approximates the radius of candidate votes in dense areas.
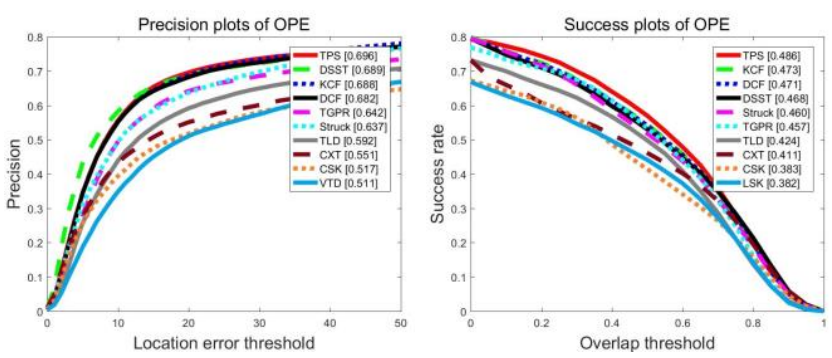

(a)
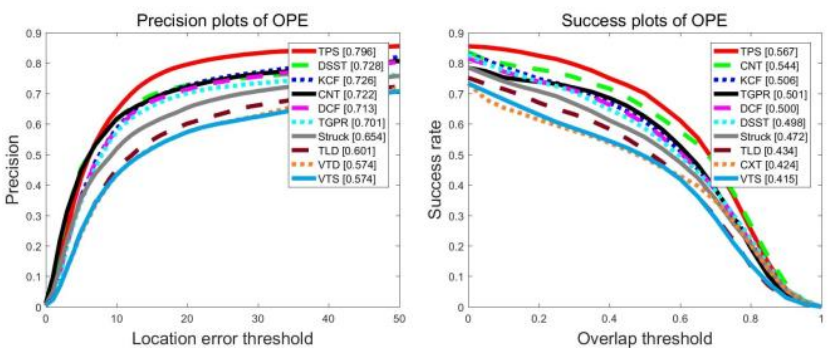

(b)

Fig. 3. Overall performance of 31 state-of-the-art trackers and our tracker on OTB-100 and CVPR2013. For clarity, only top 10 trackers are displayed. (a) Results of OPE on OTB-100. (b) Results of OPE on CVPR2013.

By normalizing the voting stability $\tau$, we obtain the acceptance ratio:

$$
\beta=\frac{\tau}{i=1} c_{i} .
$$

We denote $\beta_{l}$ as the acceptance ratio for part $l$. The vote of part $l$ on the target location is accepted at the probability $\beta_{l}$. To avoid the situation that no parts are accepted, we set a minimum number as $L_{\min }=3$. When the number of accepted parts $L_{\beta}$ is less than $L_{\min }$, we repeat the process until it is larger than $L_{\min }$.

\section{Locating and Relocating}

With $L_{\beta}$ accepted parts (denote the indexes as $p_{1}, \cdots, p_{L_{\beta}}$ ) and their estimated states $\left\{\hat{\mathbf{v}}^{\left(p_{i}\right)}\right\}_{i=1}^{L_{\beta}}$, their votes to the target can be obtained with the part offsets:

$$
\hat{\mathbf{s}}_{p}=\hat{\mathbf{v}}^{\left(p_{i}\right)}+\sigma \mathbf{v}^{\left(p_{i}\right)} \text {, }
$$

where $\sigma \mathbf{v}^{\left(p_{i}\right)}=\mathbf{s}-\mathbf{v}^{\left(p_{i}\right)}$ denotes the offset between the target state $\mathbf{s}$ and the groundtruth location of part $p_{i}$, and it is calculated in the first frame. Similar to Section III-C, we calculate the weight for each vote $w_{i}$ with the dominant set algorithm [28]. Finally, the target is located as:

$$
\hat{\mathbf{s}}={ }_{i=1}^{L \beta} w_{i} \hat{\mathbf{s}_{p_{i}}}
$$

For the unaccepted parts (denote the indexes as $q_{1}, \cdots, q L_{q}$ where $L_{q}=L-L_{\beta}$ ), we need to relocate them according to the estimated target location in the current frame.

First, we pull these parts to the corresponding anchor points on the target:

$$
\mathbf{v}_{0}^{\left(q_{i}\right)}=\hat{\mathbf{s}}-6 \mathbf{v}^{\left(q_{i}\right)}, \quad i=1, \cdots, L_{q} .
$$



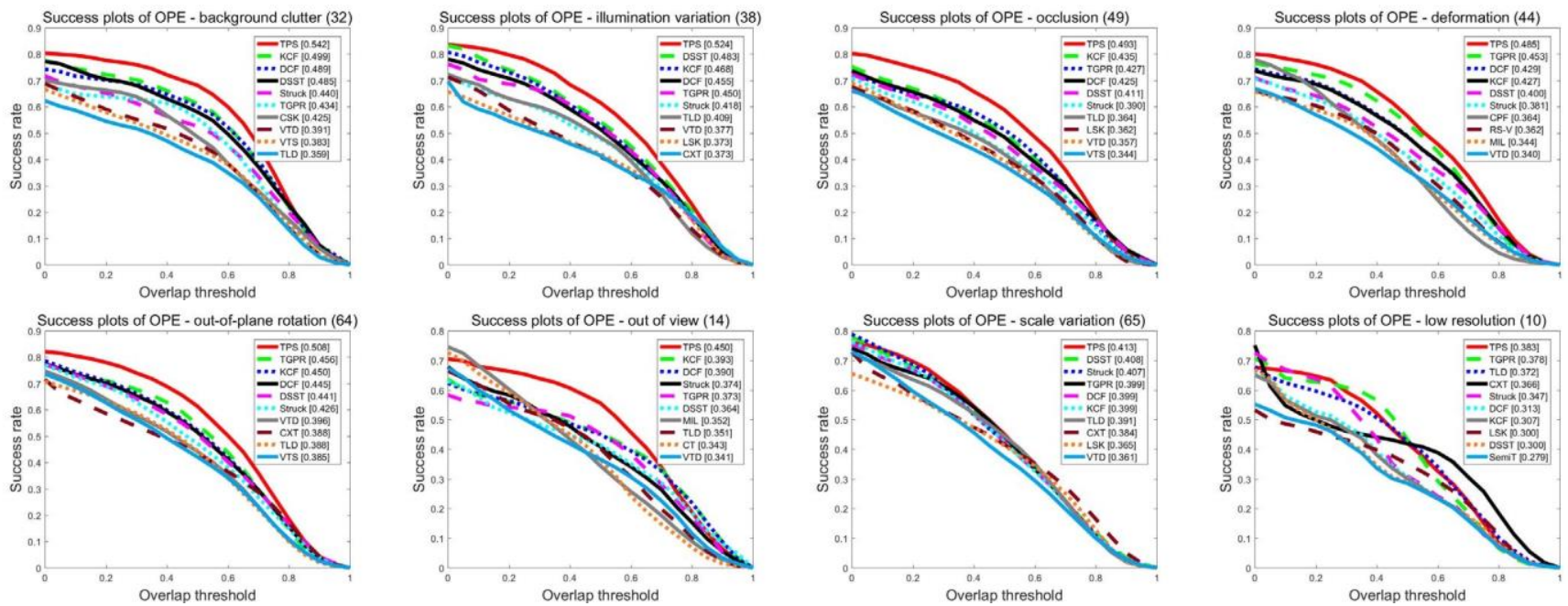

Fig. 4. The success plots of videos with different attributes on OTB-100. The number in the title indicates the number of sequences.

Then, for each part, starting from $\mathbf{v}^{\left(q_{i}\right)}$ we locate it with its observation model as described in Section III-C to obtain the estimated state $\tilde{\mathbf{v}}^{\left(q_{i}\right)}$. Denote $\rho_{i}=x_{\mathbf{v}}{ }_{0}^{\left(q_{i}\right)}-\hat{\mathbf{v}}{ }^{\left(q_{i}\right)} x_{2}^{2}$ as the Euclidean distance between $\mathbf{v}_{0}^{\left(q_{i}\right)}$ and $\tilde{\mathbf{v}}^{\left(q_{i}\right)}$. We set the final relocated part state as:

$$
\hat{\mathbf{v}}\left(q_{i}\right)=\begin{gathered}
\mathbf{v}^{\left(q_{i}\right)} \\
\mathbf{v}^{\left(q_{i}\right)} \quad \rho_{i} \leq \zeta, \\
0, \quad \rho_{i}>\zeta,
\end{gathered}
$$

where $\zeta$ is a threshold setting to 15 pixels in our experiments.

Algorithm 1 summarizes our tracking method in part space.

\section{EXPERIMENTS}

We abbreviate our method as TPS, which is short for Tracking by sampling in Part Space. To demonstrate the effectiveness of the proposed method, the TPS is evaluated on two popular benchmarks: OTB-100 [31] with 100 sequences and CVPR2013 [1], which is a subset containing 51 challenging sequences, and compared with 31 trackers, 28 of which are recommended by [1] including Struck [4], Sparsity-based Collaborative Model (SCM) [7], TrackingLearning-Detection (TLD) [32], Visual Tracking Decomposition (VTD) [33] and Compressive Tracking (CT) [34], while Discriminative Correlation Filters (DCF) [36], Kernelized Correlation Filters (KCF) [6], Discriminative Scale Space Tracker (DSST) [37], Transfer learning tracker with Gaussian Processes Regression (TGPR) [40] and Convolutional Network Tracking (CNT) [2] are recent state-of-the-art trackers, and Tracking by Regression with Incrementally Learned Cascades (TRIC) [44] is a part-based tracking method.

\section{A. Implementation Details}

Sampled image patches for each part are converted to grayscale and normalized to $32 \times 32$, and then the improved HOG feature [14] is extracted on it with bin width 4 . For simplicity, we only estimate the target's central coordinates $\mathbf{s}=\{x, y\}$ and assume the scale and angle of the target stay the same throughout the tracking process. In training and updating stage, we sample 200 images around the estimated position for each part with sample radius $r_{1}=8$. We train $C=3$ cascades of SVR with these samples. The regularization parameters are set as $\eta_{1}=0.001, \eta_{2}=0.001 . \varepsilon_{1}$ and $\varepsilon_{2}$ are fixed to 5 and 1 respectively, while $\sigma_{1}$ and $\sigma_{2}$ are fixed to 4 and 3 respectively. In the testing stage, 400 images are sampled for each part around its last estimated location with sample radius $r_{2}=20$. The model updating for each part is performed each time when $T=5$ frames of training data are collected, while the updating of the probabilities $\boldsymbol{\alpha}$ and $\boldsymbol{\beta}$ is performed in every frame. All the above parameters are fixed for fair comparison.

\section{B. Quantitative Evaluation}

1) Evaluation Criteria: The precision and success plots [1] are applied to evaluate the robustness of trackers. The precision plot indicates the percentage of frames whose estimated location is within the given threshold distance to the ground truth. The success plot demonstrates the ratios of successful frames whose overlap rate is larger than the given threshold. The precision score is given by the score on a selected threshold (e.g., 20 pixels). The success score is evaluated by the area under curve (AUC) of each tracker. For clarity, only top 10 trackers are illustrated on both plots.

2) Overall Performance: The overall performances of the 31 trackers and our tracker are shown in Fig. 3. For the precision plot, the results at error threshold of 20 pixels are used for ranking, and for the success plot we use AUC scores to rank the trackers. The performance score of each tracker is shown in the legend of Fig. 3. For OTB-100, in the precision plot, our tracker outperforms DSST by $1 \%$ and outperforms $\mathrm{KCF}$ by $1.4 \%$. In the success plot, our tracker performs $2.7 \%$ better than $\mathrm{KCF}$ and $3 \%$ better than DCF. For CVPR2013 dataset, our tracker outperforms DSST by $8.4 \%$ and outperforms $\mathrm{KCF}$ by $8.8 \%$ in terms of the precision score. In the success plot, our tracker achieves the AUC of 0.567 , which performs $4 \%$ better than CNT and $10.8 \%$ better than KCF. Overall, our tracker outperforms the state- ofthe-art trackers in terms of location accuracy and overlap precision. 


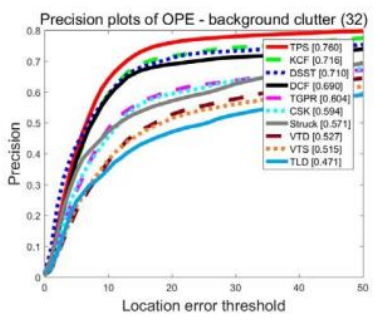

Precision plots of OPE - out-of-plane rotation (64)

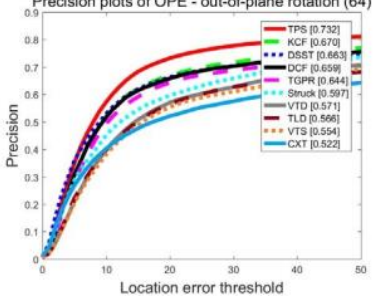

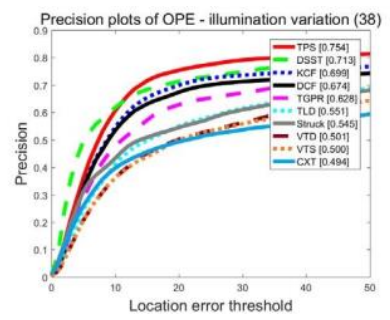

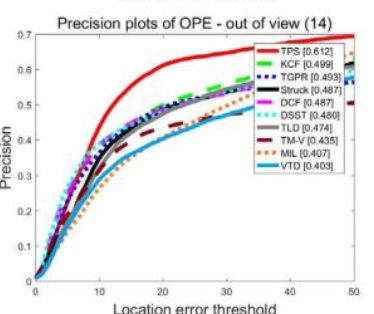

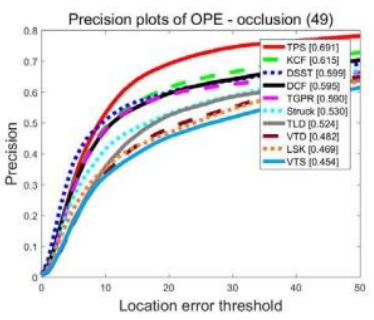

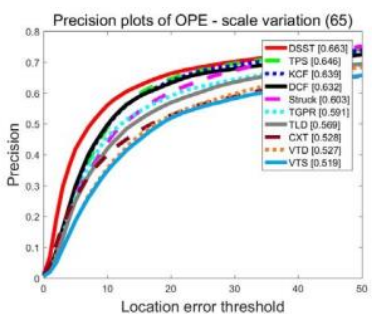

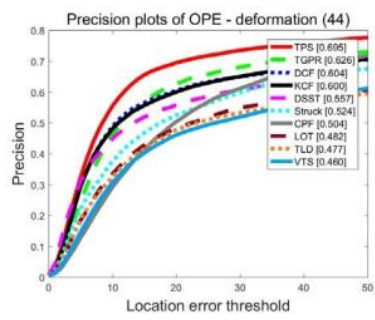

Precision plots of OPE - low resolution (10)

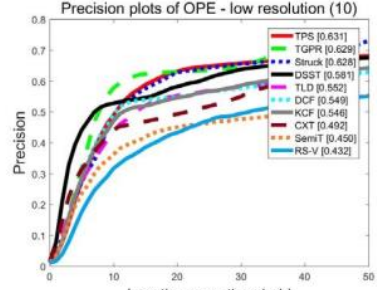

Fig. 5. Precision plots of videos with different attributes on OTB-100. The number in the title indicates the number of sequences.

TABLE I

Per-Video Precision Scores on 14 Selected Sequences. The Best Results Are Reported in Bold

\begin{tabular}{|c|c|c|c|c|c|c|c|c|c|c|c|c|c|c|}
\hline & Box & DragonBaby & Gym & Board & Human9 & $\overline{\text { KiteSurf }}$ & Panda & Tiger2 & Skater2 & Girl2 & Couple & Basketball & Football & Soccer \\
\hline Ours & 0.87 & 0.59 & 0.96 & 0.86 & 1.00 & 1.00 & 1.00 & 0.88 & 0.69 & 0.39 & 1.00 & 0.94 & 0.99 & 0.85 \\
\hline DSST & 0.39 & 0.06 & 0.78 & 0.77 & 0.37 & 0.42 & 0.19 & 0.28 & 0.37 & 0.07 & 0.11 & 0.79 & 0.79 & 0.60 \\
\hline $\mathrm{KCF}$ & 0.42 & 0.34 & 0.77 & 0.62 & 0.67 & 0.74 & 0.33 & 0.34 & 0.68 & 0.07 & 0.26 & 0.92 & 0.80 & 0.78 \\
\hline TLD & 0.74 & 0.21 & 0.83 & 0.08 & 0.22 & 0,33 & 0.99 & 0.35 & 0.46 & 0.25 & 0.31 & 0.51 & 0.79 & 0.10 \\
\hline Struck & 0.71 & 0.08 & 0.10 & 0.82 & 0,08 & 0,23 & 1.00 & 0.38 & 0.64 & 0.07 & 0.82 & 0.02 & 0.69 & 0.18 \\
\hline
\end{tabular}

TABLE II

Per-Video Success Scores on 14 Selected Sequences. The Best Results Are Reported in Bold

\begin{tabular}{ccccccccccccccccc}
\hline & Box & DragonBaby & Gym & Board & Human9 & KiteSurf & Panda & Tiger2 & Skater2 & Girl2 & Couple & Basketball & Football & Soccer \\
\hline Ours & $\mathbf{0 . 6 7}$ & $\mathbf{0 . 4 4}$ & $\mathbf{0 . 4 9}$ & $\mathbf{0 . 7 5}$ & $\mathbf{0 . 3 9}$ & $\mathbf{0 . 7 0}$ & $\mathbf{0 . 5 8}$ & $\mathbf{0 . 7 1}$ & $\mathbf{0 . 5 9}$ & $\mathbf{0 . 2 8}$ & $\mathbf{0 . 6 5}$ & $\mathbf{0 . 7 2}$ & $\mathbf{0 . 7 5}$ & $\mathbf{0 . 4 5}$ \\
DSST & 0.35 & 0.06 & 0.25 & 0.72 & 0.32 & 0.33 & 0.12 & 0.33 & 0.42 & 0.09 & 0.09 & 0.58 & 0.56 & 0.44 \\
KCF & 0.30 & 0.31 & 0.43 & 0.66 & $\mathbf{0 . 3 9}$ & 0.48 & 0.16 & 0.35 & 0.57 & 0.06 & 0.20 & 0.68 & 0.55 & 0.42 \\
TLD & 0.42 & 0.15 & 0.44 & 0.22 & 0.17 & 0.23 & 0.54 & 0.29 & 0.40 & 0.17 & 0.19 & 0.31 & 0.54 & 0.12 \\
Struck & 0.55 & 0.13 & 0.08 & 0.73 & 0.07 & 0.19 & 0.53 & 0.35 & 0.57 & 0.06 & 0.53 & 0.02 & 0.54 & 0.15 \\
\hline
\end{tabular}

TABLE III

Performance Improvement of Different Subsets in Terms of Precision and Success SCORES COMPARED With THE SECOND-RANKed TRACKERS

\begin{tabular}{ccccccccc}
\hline & OCC & OV & OPR & BC & IV & DEF & SV & LR \\
\hline Precision Score & $+5.8 \%$ & $+5.7 \%$ & $+5.2 \%$ & $+4.4 \%$ & $+4.1 \%$ & $+3.2 \%$ & $+0.5 \%$ & $+0.5 \%$ \\
Success Score & $+7.6 \%$ & $+11.3 \%$ & $+6.2 \%$ & $+4.3 \%$ & $+4.1 \%$ & $+6.9 \%$ & $-1.7 \%$ & $+0.2 \%$ \\
\hline
\end{tabular}

3) Attribute-Based Performance: Several factors can affect the performance of an object tracker. In the OTB-100 dataset, the 100 sequences are annotated with different challenging attributes that may affect tracking performance, such as occlusion, background clutters, object deformation. Fig. 4 and Fig. 5 show the success plots and precision plots of 31 state-of- theart trackers and our tracker on 8 different video subsets. In addition, Table I and Table II also illustrate the perfor- mance of our tracker and other four state-of-the-art methods on 14 selected challenging videos. The Box, DragonBaby, KiteSurf, Panda, Tiger2, Basketball, Football and Soccer are selected from the Occlusion subset, while the Gym, Panda, Human9, Skater2, Girl2 and Couple are selected from the
Deformation subset. In addition, the sequences Box, DragonBaby, Gym, Board, Human9, Panda, Skater2, Girl2, Couple and Soccer also belong to the Scale Variation subset, and the sequences Basketball, Board, Couple, Football and Soccer also belong to the Background Clutter subset.

Though our tracker only estimates the center location and does not predict scales, it achieves comparable or even better results than other methods (e.g. DSST) on the Scale Variations subset. This is because the large correlation among different attributes. As shown in Table I and Table II, the sequences Box, DragonBaby, Human9, Girl2, Panda, Skater2 and Couple belong to the Scale Variations subset, but the objects also suffer from occlusions, background clutter and object 

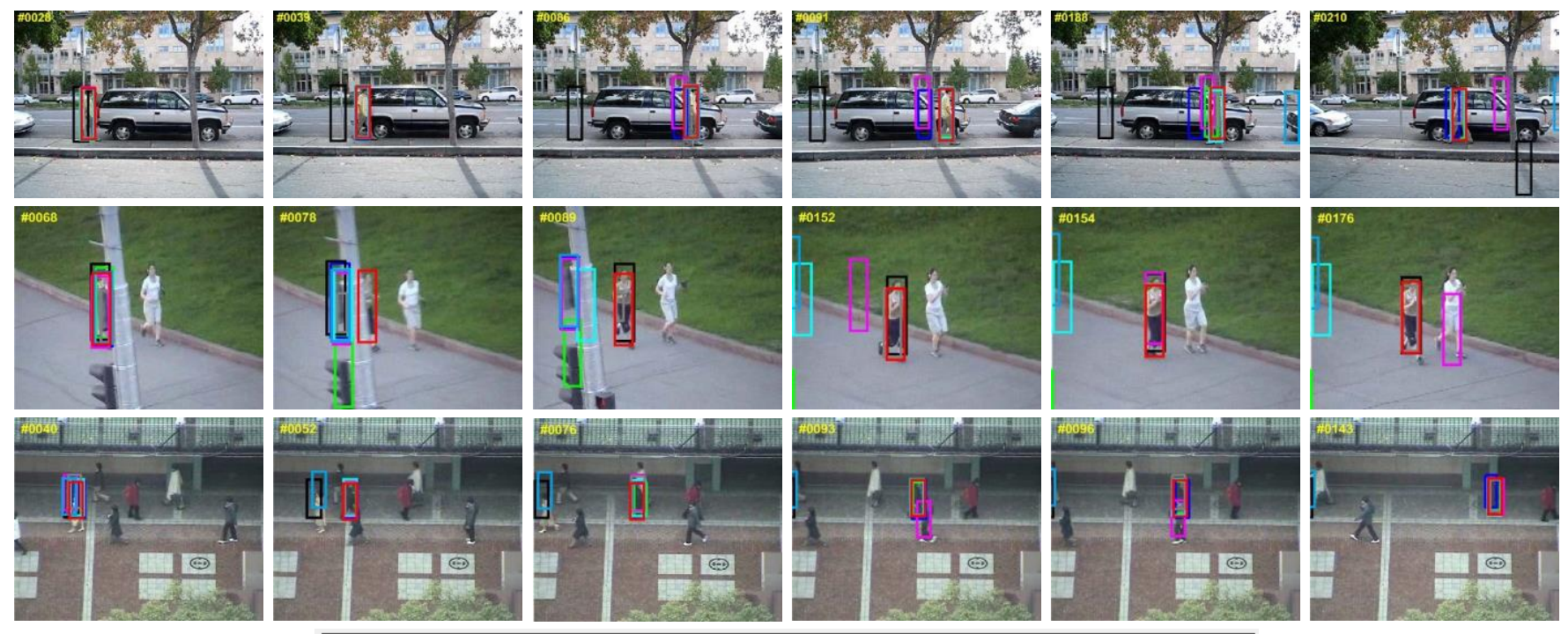

$-\mathrm{TPS}=\mathrm{TGPR}=\mathrm{SCM}-\mathrm{TLD}=\mathrm{Struck}-\mathrm{KCF}=\mathrm{CNT}=\mathrm{DSST}$

Fig. 6. From top to bottom are representative results of trackers on sequences David3, Jogging-1 and Subway, where objects are heavily occluded.

deformation. Though previous trackers can estimate scales very well, they fail to track these clips, while our method performs much better in tracking occluded or deformed objects.

To better illustrate the pros and cons of our method, we rank the improvement of performance in different subsets according to the precision scores and list them in Table III. As shown in Table III, the main improvement of performance come from the Occlusions, Out-of-View, Out-of-plane Rotation, Background Clutter, Illumination Variation and Deformation subsets. Our tracker achieves better performance on the Occlusion and Deformation subsets, which validates the effectiveness of the proposed part-based model. It effectively selects and

combines different parts to obtain stable results. The good performance of our method on the Out-of-view, Out-of-plane Rotation and Background Clutter subsets could be attributed to our voting process. It considers location estimations from multiple surrounded candidates and locates the target with

the combination of these votes. It also can successfully locate the target when some of the surrounded candidates are invisible (e.g., occluded or out-of-view) or interrupted by the background noise.

\section{Qualitative Evaluation}

Now we present a qualitative evaluation of the tracking results. 12 representative sequences with different challenges are selected from the 100 sequences in OTB-100. The three dominant challenges of these sequences are occlusion, object deformation, and illumination variation. Fig. 6 - Fig. 8 show some screenshots of the tracking results of our tracker and some competitive state-of-the art trackers.

1) Occlusion: Occlusion is one of the most critical challenges in visual tracking. Fig. 6 illustrates tracking results on three representative sequences (David3, Jogging-1 and Subway) where objects are severely or long-term occluded. In the David3 sequence, David is completely occluded several times by the pole and the tree (e.g., \#28, \#91). TLD, SCM and Struck fail to re-detect the target when David reappears in the screen. Our method, KCF, CNT and DSST achieve favorable results. In the Jogging-1 sequence, the left girl is occluded fully by the telegraph pole (e.g., \#68, \#78). Only our method, CNT, TGPR and TLD can track the target successfully (e.g., \#89, \#152, \#176). In sequence Subway, a person is occluded by other people in some frames (e.g., \#41, \#96). Only TPS, TGPR, SCM and KCF are able to track the target stably. Note that KCF updates with an exponential decay factor. Thus it can deal with short-term occlusions while long-term occlusions make it drift to the background. The superior performance of our method could be attributed to the part-based model. The proposal distribution helps selecting stable parts for tracking while the acceptance ratio avoids the bounding box drifting to the occluded parts.

2) Object Deformation: In Fig. 7, sequences Panda and Singer2 are selected to show the robustness of trackers against non-rigid object deformation. The target in the Singer 2 sequence has significant appearance variations due to illumination changes and non-rigid body deformation. Struck, SCM, TGPR and TLD fail to track the target (e.g., \#22, \#78, \#135). Our method performs well at all frames. The target in the Panda sequence walks around the screen all the time, which makes it undergo both deformation and occlusion. KCF, TLD and SCM lose the target in the tracking process (e.g., \#315, \#590, \#686). The holistic models, i.e., Struck, TLD, KCF and TGPR have difficulty in tracking non-rigid objects while SCM uses a weighted updating strategy, making it prone to drift to the background. Our method performs well in the whole sequence for two reasons. The part-based models are skilled in tracking non-rigid objects while the proposed online SVR provides an elegant way to incorporate previous model with new observations.

3) Illumination Variation: Fig. 8 shows tracking results on two challenging clips (Sylvester and Skating1), where objects 

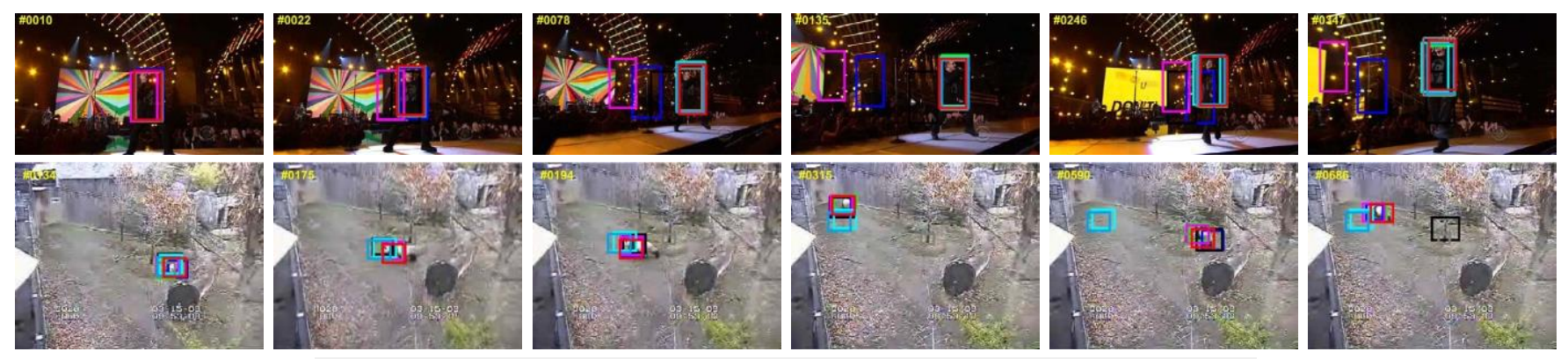

$=$ TPS $=$ TGPR $=$ SCM - TLD $=$ Struck $=$ KCF $=$ CNT $=\mathrm{DSST}$

Fig. 7. From top to bottom are representative results on sequences Singer2 and Panda. Object deformation is the main challenge of these sequences.
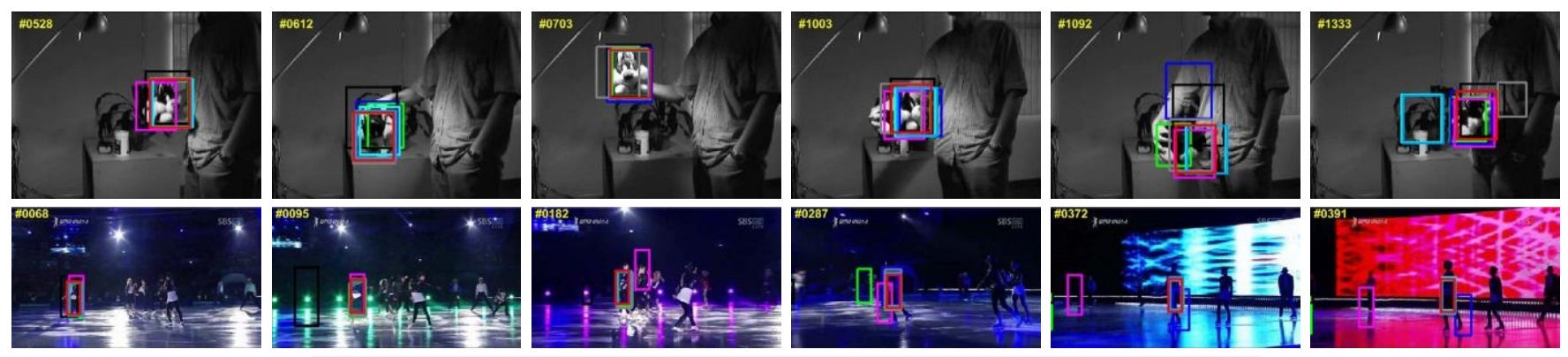

$-\mathrm{TPS}=\mathrm{TGPR}=\mathrm{SCM}-\mathrm{TLD}=\mathrm{Struck}=\mathrm{KCF}=\mathrm{CNT}=\mathrm{DSST}$

Fig. 8. From top to bottom are representative results on sequences Sylvester and Skating1, where objects suffer from illumination variations.

undergo significant illumination changes. In the Sylvester sequence, a doll moves quickly under the light. Despite heavy illumination variations in some frames (e.g., \#528, \#612, \#703), our method is able to track the target well. Struck, TLD, CNT and KCF lose the target when sudden illumination changes and fast motion occur (e.g., \#1003, \#1092, \#1333). When the target glides on the ice in sequence Skatingl, it undergoes severe deformation and dramatic light changes (e.g., \#68, \#182). Only our method, CNT, SCM and $\mathrm{KCF}$ can track the target from the beginning to the end. The promising tracking results of our tracker on the illumination subset could be attributed to the improved HOG feature [14] used in our method, which is invariant to local illumination variations.

\section{CONCLUSIONS}

We have presented a part-based tracking method from the perspective of probability sampling. Our tracking model is constructed by a triplet: a part space and two probabilities - the proposal distribution and the acceptance probability on it. The proposal distribution is learned online to capture the structure and appearance of the target, while the acceptance probability is calculated to determine the credibility of the tracking result of each part. For learning and updating the appearance model of each part online, we have developed an incremental cascaded support vector regression algorithm. Three components are united for the construction of the observation model for robustly tracking against local appearance variations. Experimental results on two recent benchmarks have demonstrated the superior performance of our method.

\section{REFERENCES}

[1] Y. Wu, J. Lim, and M.-H. Yang, "Online object tracking: A benchmark," in Proc. IEEE Conf. Comput. Vis. Pattern Recognit., Jun. 2013, pp. 2411-2418.

[2] K. Zhang, Q. Liu, Y. Wu, and M.-H. Yang, "Robust visual tracking via convolutional networks without training," IEEE Trans. Image Process., vol. 25, no. 4, pp. 1779-1792, Apr. 2016.

[3] J. Zhang, S. Ma, and S. Sclaroff, "MEEM: Robust tracking via multiple experts using entropy minimization," in Proc. Eur. Conf. Comput. Vis., 2014, pp. 188-203.

[4] S. Hare, A. Saffari, and P. H. S. Torr, "Struck: Structured output tracking with kernels," in Proc. IEEE Int. Conf. Comput. Vis., Nov. 2011, pp. 263-270.

[5] F. Yang, H. Lu, and M.-H. Yang, "Robust superpixel tracking," IEEE Trans. Image Process., vol. 23, no. 4, pp. 1639-1651, Apr. 2014.

[6] J. F. Henriques, R. Caseiro, P. Martins, and J. Batista, "High-speed tracking with kernelized correlation filters," IEEE Trans. Pattern Anal. Mach. Intell., vol. 37, no. 3, pp. 583-596, Mar. 2015.

[7] W. Zhong, H. Lu, and M.-H. Yang, "Robust object tracking via sparsitybased collaborative model," in Proc. IEEE Conf. Comput. Vis. Pattern Recognit., Jun. 2012, pp. 1838-1845.

[8] X. Mei and H. Ling, "Robust visual tracking using 41 minimization," in Proc. IEEE Int. Conf. Comput. Vis., Sep. 2009, pp. 1436-1443.

[9] T. Zhang, B. Ghanem, S. Liu, and N. Ahuja, "Robust visual tracking via multi-task sparse learning," in Proc. IEEE Conf. Comput. Vis. Pattern Recognit., Jun. 2012, pp. 2042-2049.

[10] Q. Wang, F. Chen, J. Yang, W. Xu, and M.-H. Yang, "Transferring visual prior for online object tracking," IEEE Trans. Image Process., vol. 21, no. 7, pp. 3296-3305, Jul. 2012.

[11] B. Ma, L. Huang, J. Shen, and L. Shao, "Discriminative tracking using tensor pooling," IEEE Trans. Cybern., vol. 46, no. 11, pp. 2411-2422, Nov. 2015.

[12] R. Yao, Q. Shi, C. Shen, Y. Zhang, and A. van den Hengel, "Part-based visual tracking with online latent structural learning," in Proc. IEEE Conf. Comput. Vis. Pattern Recognit., Jun. 2013, pp. 2363-2370.

[13] A. Smith, A. Doucet, N. de Freitas, and N. Gordon, Sequential Monte Carlo Methods in Practice. New York, NY, USA: Springer, 2013. 
[14] P. F. Felzenszwalb, R. B. Girshick, D. McAllester, and D. Ramanan, "Object detection with discriminatively trained part-based models," IEEE Trans. Pattern Anal. Mach. Intell., vol. 32, no. 9, pp. 1627-1645, Sep. 2010.

[15] P. F. Felzenszwalb, R. B. Girshick, and D. McAllester, "Cascade object detection with deformable part models," in Proc. IEEE Conf. Comput. Vis. Pattern Recognit., Jun. 2010, pp. 2241-2248.

[16] H. Azizpour and I. Laptev, "Object detection using strongly-supervised deformable part models," in Proc. Eur. Conf. Comput. Vis., 2012, pp. 836-849.

[17] Y. Tian, R. Sukthankar, and M. Shah, "Spatiotemporal deformable part models for action detection," in Proc. IEEE Conf. Comput. Vis. Pattern Recognit., Jun. 2013, pp. 2642-2649.

[18] X. Xiong and F. de la Torre, "Supervised descent method and its applications to face alignment," in Proc. IEEE Conf. Comput. Vis. Pattern Recognit., Jun. 2013, pp. 532-539.

[19] X. Song, T. Wu, Y. Jia, and S.-C. Zhu, "Discriminatively trained andor tree models for object detection," in Proc. IEEE Conf. Comput. Vis. Pattern Recognit., Jun. 2013, pp. 3278-3285.

[20] Y. Lu, T. Wu, and S. C. Zhu, "Online object tracking, learning, and parsing with and-or graphs," in Proc. IEEE Conf. Comput. Vis. Pattern Recognit., Jun. 2014, pp. 3462-3469.

[21] M. D. Breitenstein, F. Reichlin, B. Leibe, E. Koller-Meier, and L. Van Gool, "Robust tracking-by-detection using a detector confidence particle filter," in Proc. IEEE Int. Conf. Comput. Vis., Sep. 2009, pp. 1515-1522.

[22] J. Shen, Y. Du, W. Wang, and X. Li, "Lazy random walks for superpixel segmentation," IEEE Trans. Image Process., vol. 23, no. 4, pp. 1451-1462, Apr. 2014.

[23] X. Jia, H. Lu, and M.-H. Yang, "Visual tracking via adaptive structural local sparse appearance model," in Proc. IEEE Conf. Comput. Vis. Pattern Recognit., Jun. 2012, pp. 1822-1829.

[24] D. A. Ross, J. Lim, R.-S. Lin, and M.-H. Yang, "Incremental learning for robust visual tracking," Int. J. Comput. Vis., vol. 77, nos. 1-3, pp. 125-141, 2008.

[25] X. Xiong and F. de la Torre. (2014). "Supervised descent method for solving nonlinear least squares problems in computer vision." [Online]. Available: https://arxiv.org/abs/1405.0601

[26] W. Wang, J. Shen, X. Li, and F. Porikli, "Robust video object cosegmentation," IEEE Trans. Image Process., vol. 24, no. 10, pp. 3137-3148, Oct. 2015.

[27] X. Xiong and F. De la Torre, "Global supervised descent method," in Prco. IEEE Conf. Comput. Vis. Pattern Recognit., Jun. 2015, pp. 2664 2673.

[28] M. Pavan and M. Pelillo, "Dominant sets and pairwise clustering," IEEE Trans. Pattern Anal. Mach. Intell., vol. 29, no. 1, pp. 167-172, Jan. 2007.

[29] W. Wang, J. Shen, and F. Porikli, "Saliency-aware geodesic video object segmentation," in Proc. IEEE Conf. Comput. Vis. Pattern Recognit., Jun. 2015, pp. 3395-3402.

[30] Z. Wang and S. Vucetic, "Online training on a budget of support vector machines using twin prototypes," Statist. Anal. Data Mining, vol. 3, no. 3, pp. 149-169, Jun. 2010.

[31] Y. Wu, J. Lim, and M. H. Yang, "Object tracking benchmark," IEEE Trans. Pattern Anal. Mach. Intell., vol. 37, no. 9, pp. 1834-1848, Sep. 2015.

[32] Z. Kalal, K. Mikolajczyk, and J. Matas, "Tracking-learning-detection," IEEE Trans. Pattern Anal. Mach. Intell., vol. 34, no. 7, pp. 1409-1422, Jul. 2012.

[33] J. Kwon and K. M. Lee, "Visual tracking decomposition," in Proc. IEEE Conf. Comput. Vis. Pattern Recognit., Jun. 2010, pp. 1269-1276.

[34] K. Zhang, L. Zhang, and M. H. Yang, "Real-time compressive tracking," in Proc. Eur. Conf. Comput. Vis., 2012, pp. 864-877.

[35] B. Ma, L. Huang, J. Shen, L. Shao, M.-H. Yang, and F. Porikli, "Visual tracking under motion blur," IEEE Trans. Image Process., vol. 25, no. 12 , pp. 5867-5876, Dec. 2016.

[36] J. F. Henriques, R. Caseiro, P. Martins, and J. Batista, "Exploiting the circulant structure of tracking-by-detection with kernels," in Proc. Eur. Conf. Comput. Vis., 2012, pp. 702-715.

[37] M. Danelljan, G. Häger, F. S. Khan, and M. Felsberg, "Accurate scale estimation for robust visual tracking," in Proc. Brit. Mach. Vis. Conf., 2015, pp. 1-11.
[38] J. Kwon and K. M. Lee, "Tracking by sampling trackers," in Proc. IEEE Int. Conf. Comput. Vis., Nov. 2011, pp. 1195-1202.

[39] S. Hong and B. Han, "Visual tracking by sampling tree-structured graphical models" In Proc. Eur. Conf. Comput. Vis., 2014, pp. 1-16.

[40] J. Gao, H. Ling, W. Hu, and J. Xing, "Transfer learning based visual tracking with Gaussian processes regression," in Proc. Eur. Conf. Comput. Vis., 2014, pp. 188-203.

[41] B. Ma, J. Shen, Y. Liu, H. Hu, L. Shao, and X. Li, "Visual tracking using strong classifier and structural local sparse descriptors," IEEE Trans. Multimedia, vol. 17, no. 10, pp. 1818-1828, Oct. 2015.

[42] Y. Li, J. Zhu, and S. C. Hoi, "Reliable patch trackers: Robust visual tracking by exploiting reliable patches," in Proc. IEEE Conf. Comput. Vis. Pattern Recognit., Jun. 2015, pp. 353-361.

[43] B. Ma, H. Hu, J. Shen, Y. Liu, and L. Shao, "Generalized pooling for robust object tracking," IEEE Trans. Image Process., vol. 25, no. 9, pp. 4199-4208, Sep. 2016.

[44] X. Wang, M. Valstar, B. Martinez, M. H. Khan, and T. Pridmore, "TRICtrack: Tracking by regression with incrementally learned cascades," in Proc. IEEE Int. Conf. Comput. Vis., Dec. 2015, pp. 4337-4345.

[45] D. Zhang, J. Han, C. Li, J. Wang, and X. Li, "Detection of co-salient objects by looking deep and wide," Int. J. Comput. Vis., vol. 120, no. 2, pp. 215-232, Nov. 2016.

[46] B. Ma, H. Hu, J. Shen, Y. Zhang, and F. Porikli, "Linearization to nonlinear learning for visual tracking," in Proc. IEEE Int. Conf. Comput. Vis., Dec. 2015, pp. 4400-4407.

[47] D. Zhang, J. Han, J. Han, and L. Shao, "Cosaliency detection based on intrasaliency prior transfer and deep intersaliency mining," IEEE Trans. Neural Netw. Learn. Syst., vol. 27, no. 6, pp. 1163-1176, Jun. 2016.

[48] X. Dong, J. Shen, D. Yu, W. Wang, J. Liu, and H. Huang, "Occlusionaware real-time object tracking," IEEE Trans. Multimedia, vol. 19, no. 4 , pp. 763-771, Apr. 2017.

[49] T. Liu, G. Wang, and Q. Yang, "Real-time part-based visual tracking via adaptive correlation filters," in Proc. IEEE Conf. Comput. Vis. Pattern Recognit., Jun. 2015, pp. 4902-4912.

Lianghua Huang, photograph and biography not available at the time of publication.

Bo Ma, photograph and biography not available at the time of publication.

Jianbing Shen, photograph and biography not available at the time of publication.

Hui He, photograph and biography not available at the time of publication.

Ling Shao, photograph and biography not available at the time of publication.

Fatih Porikli, photograph and biography not available at the time of publication. 